\title{
Homoclinic orbits for damped vibration systems with asymptotically quadratic or subquadratic potentials
}

\author{
Huiwen Chen ${ }^{1 *}$, Zhimin $\mathrm{He}^{2}$, Zigen Ouyang ${ }^{1}$ and Maoxin Liao
}

\section{"Correspondence:}

huiwchen@163.com

${ }^{1}$ School of Mathematics and

Physics, University of South China,

Hengyang, Hunan 421001, China

Full list of author information is

available at the end of the article

\begin{abstract}
In this paper, we study a class of damped vibration systems,

$$
\ddot{u}(t)+B \dot{u}(t)-L(t) u(t)+\nabla W(t, u(t))=0, \quad \forall t \in \mathbb{R},
$$

where $W(t, u)$ is of indefinite sign. By using a critical point theorem of Ding, we establish a new criterion to guarantee that the above system has infinitely many nontrivial homoclinic orbits under the assumption that $W(t, u)$ is asymptotically quadratic or subquadratic as $|u| \rightarrow \infty$. Recent results in the literature are generalized and significantly improved.
\end{abstract}

MSC: $34 \mathrm{C} 37 ; 35 \mathrm{~A} 15 ; 37 \mathrm{~J} 45$

Keywords: damped vibration systems; homoclinic orbits; variational methods; asymptotically quadratic theorem; subquadratic

\section{Introduction}

In this paper, we consider the following damped vibration system:

$$
\ddot{u}(t)+B \dot{u}(t)-L(t) u(t)+\nabla W(t, u(t))=0, \quad \forall t \in \mathbb{R},
$$

where $u \in \mathbb{R}^{N}, B$ is an antisymmetric $N \times N$ constant matrix, $L \in C\left(\mathbb{R}, \mathbb{R}^{N \times N}\right)$ is a symmetric matrix-valued function, and $W \in C^{1}\left(\mathbb{R} \times \mathbb{R}^{N}, \mathbb{R}\right)$. As usual, we say that a solution $u$ of system (1.1) is homoclinic to zero if $u \in C^{2}\left(\mathbb{R}, \mathbb{R}^{N}\right), u(t) \rightarrow 0$, and $\dot{u}(t) \rightarrow 0$ as $|t| \rightarrow \infty$. In addition, if $u(t) \not \equiv 0$, then $u(t)$ is called a nontrivial homoclinic solution.

Homoclinic orbits have been found in various models of continuous dynamical systems and play an important role in the study of the behavior of dynamical systems; see [1]. Thus, the study of homoclinic orbits has become one of the most important directions in the research of dynamical systems.

When $B=0$, system (1.1) reduces to the following second order Hamiltonian system:

$$
\ddot{u}(t)-L(t) u(t)+\nabla W(t, u(t))=0, \quad \forall t \in \mathbb{R} .
$$

(c) 2016 Chen et al. This article is distributed under the terms of the Creative Commons Attribution 4.0 International License (http://creativecommons.org/licenses/by/4.0/), which permits unrestricted use, distribution, and reproduction in any medium, provided you give appropriate credit to the original author(s) and the source, provide a link to the Creative Commons license, and indicate if changes were made. 
As a special case of dynamical systems, Hamiltonian systems play an important role in practical problems concerning relativistic mechanics, gas dynamics, nuclear physics, fluid mechanics. With the aids of the variational methods, the existence and multiplicity of homoclinic orbits for (1.2) have been extensively investigated in many recent papers; see [2-24].

Compared with the case where $B=0$, the case where $B \neq 0$ is more difficult. The study of homoclinic orbits for system (1.1) has attracted a lot of attention by many researchers; see [25-33]. This work is mainly based on variational methods. Some of the authors considered the superquadratic case $[27,29,30,32,33]$; the authors of $[25,28,31]$ considered the subquadratic case; for the asymptotically quadratic case, except for [26], few researchers have investigated this case. More precisely, in [25], Chen studied system (1.1) under the assumption that $W(t, u)$ is subquadratic as $|u| \rightarrow \infty$. In detail, he obtained the following result.

Theorem 1.1 ([25]) Assume that the following conditions hold.

$\left(\mathrm{L}_{1}\right)$ There exists a constant $v>1$ such that

$$
\text { meas }\left\{t \in \mathbb{R}:|t|^{-v} L(t)<T I_{N}\right\}, \quad \forall T>0,
$$

where $I_{N}$ denotes the $N \times N$ identity matrix.

$\left(\mathrm{L}_{2}\right)$ There exists a constant $\beta \geq 0$ such that

$$
l(t):=\inf _{|u|=1}(L(t) u, u) \geq-\beta, \quad t \in \mathbb{R} .
$$

$\left(\mathrm{H}_{1}\right) W(t, u) \geq 0, \forall(t, u) \in \mathbb{R} \times \mathbb{R}^{N}$, and there exist constants $\kappa \in(0,2)$ and $R_{0}>0$ such that

$$
(\nabla W(t, u), u) \leq \kappa W(t, u), \quad \forall t \in \mathbb{R} \text { and }|u| \geq R_{0}
$$

and

$$
(\nabla W(t, u), u) \leq 2 W(t, u), \quad \forall t \in \mathbb{R} \text { and }|u| \leq R_{0} .
$$

$\left(\mathrm{H}_{2}\right)$ There exists $a>0$ such that

$$
W(t, u) \leq a|u|, \quad \forall t \in \mathbb{R} \text { and }|u| \leq R_{0} .
$$

$\left(\mathrm{H}_{3}\right) \liminf _{|u| \rightarrow \infty} \frac{W(t, u)}{|u|} \geq$ b uniformly in $t \in \mathbb{R}$, where $b>0$ is a constant.

$\left(\mathrm{H}_{4}\right) \lim _{|u| \rightarrow 0} \frac{W(t, u)}{|u|^{2}}=+\infty$ uniformly in $t \in \mathbb{R}$.

$\left(\mathrm{H}_{5}\right) W(t,-u)=W(t, u), \forall(t, u) \in \mathbb{R} \times \mathbb{R}^{N}$.

Then system (1.1) has infinitely many nontrivial homoclinic orbits.

In [26], $W(t, u)$ being asymptotically quadratic as $|u| \rightarrow \infty$, by using the variant fountain theorem, Chen obtained the following result.

Theorem $1.2([26])$ Assume that $\left(\mathrm{L}_{1}\right),\left(\mathrm{L}_{2}\right)$, and $\left(\mathrm{H}_{5}\right)$ hold. Moreover, we assume that the following conditions are satisfied: 
$\left(\mathrm{H}_{6}\right) \quad \widetilde{W}(t, u)=W(t, u)-(1 / 2)(\nabla W(t, u), u) \rightarrow+\infty$ as $|u| \rightarrow \infty$ uniformly in $t \in \mathbb{R}$.

$\left(\mathrm{H}_{7}\right)$ There are constants $\tau \in(1,2)$ and $a_{1}, a_{2}, a_{2}>0$ such that

$$
a_{3}|u|^{\tau} \leq W(t, u) \leq a_{1}|u|, \quad \forall t \in \mathbb{R} \text { and }|u| \leq a_{2} .
$$

$\left(\mathrm{H}_{8}\right) \quad \lim \sup _{|u| \rightarrow 0} \frac{\mid \nabla W(t, u)^{\tau /(\tau-1)}}{\overleftarrow{W}(t, u)}=P(t)$ uniformly in $t,|P(t)|<\infty$.

$\left(\mathrm{H}_{9}\right) \quad W(t, u) \geq(1 / 2)(\nabla W(t, u), u) \geq 0, \forall(t, u) \in \mathbb{R} \times \mathbb{R}^{N}$.

$\left(\mathrm{H}_{10}\right) \lim _{|u| \rightarrow \infty} \frac{W(t, u)}{|u|^{2}}=f(t)$ uniformly in $t$, where $\inf _{t \in \mathbb{R}} f(t) \leq \sup _{t \in \mathbb{R}} f(t)<+\infty$.

Then system (1.1) has infinitely many nontrivial homoclinic orbits.

Motivated by the above facts, in this paper, our aim is to generalize some results in $[25,26]$. Moreover, our approach is different from $[25,26]$.

We will use the following conditions:

$\left(\mathrm{W}_{1}\right) \nabla W(t, u)=S(t) u+\nabla G(t, u)$, where $S: \mathbb{R} \rightarrow \mathbb{R}^{N \times N}$ is bounded symmetric $N \times N$ matrix-valued function.

$\left(W_{2}\right) W(t, 0)=G(t, 0)=0$ and there exist $\bar{a}, \bar{b}>0$ and $0<v<1$ such that

$$
|\nabla G(t, u)| \leq \bar{a}+\bar{b}|u|^{\nu}, \quad \forall(t, u) \in \mathbb{R} \times \mathbb{R}^{N} .
$$

$\left(\mathrm{W}_{3}\right) \widetilde{W}(n, u) \geq 0, \forall(t, u) \in \mathbb{R} \times \mathbb{R}^{N}$.

Now, we state our main result.

Theorem 1.3 Assume that $\left(\mathrm{L}_{1}\right),\left(\mathrm{L}_{2}\right),\left(\mathrm{H}_{4}\right)-\left(\mathrm{H}_{6}\right),\left(\mathrm{W}_{1}\right)-\left(\mathrm{W}_{3}\right)$ hold. Then system (1.1) has infinitely many nontrivial homoclinic orbits.

Remark 1.1 When $S \not \equiv 0$, Theorem 1.3 generalizes Theorem 1.2. First of all, we remove conditions $\left(\mathrm{H}_{8}\right)$ and $W(t, u) \geq 0$ for any $(t, u) \in \mathbb{R} \times \mathbb{R}^{N}$. Second, condition $\left(\mathrm{H}_{4}\right)$ is weaker than $\left(\mathrm{H}_{7}\right)$. In fact, condition $\left(\mathrm{H}_{7}\right)$ implies $\left(\mathrm{H}_{4}\right)$. Third, it is clear that condition $\left(\mathrm{W}_{3}\right)$ is weaker than $\left(\mathrm{H}_{9}\right)$. Furthermore, there are many functions satisfying our Theorem 1.3 and not satisfying Theorem 1.2. For example, let $W(t, u)=g(t)|u|^{2}+|u|$ for all $(t, u) \in \mathbb{R} \times \mathbb{R}^{N}$, where $g: \mathbb{R} \rightarrow \mathbb{R}$ is bounded continuous function such that $g \not \equiv 0$. It is easy to check that $W(t, u)$ satisfies all conditions of Theorem 1.3, but it does not satisfy condition $\left(\mathrm{H}_{8}\right)$ of Theorem 1.2.

Remark 1.2 When $S \equiv 0$, Theorem 1.3 generalizes Theorem 1.1. First, conditions $\left(\mathrm{H}_{1}\right)$ and $\left(\mathrm{H}_{3}\right)$ imply conditions $\left(\mathrm{H}_{6}\right)$ and $\left(\mathrm{W}_{3}\right)$. Second, conditions $\left(\mathrm{H}_{1}\right)$ and $\left(\mathrm{H}_{2}\right)$ imply $\left(\mathrm{W}_{2}\right)$. Third, we remove conditions $\left(\mathrm{H}_{3}\right)$ and $W(t, u) \geq 0$ for any $(t, u) \in \mathbb{R} \times \mathbb{R}^{N}$. Furthermore, there are many functions $W(t, u)$ satisfying our Theorem 1.3 and not satisfying Theorem 1.1. For example, set

$$
W(t, u)= \begin{cases}\frac{3}{2}|u|-|u|^{\frac{3}{2}}, & |u|<1, \\ \frac{u^{2}}{2(|u|+\ln |u|)}, & |u| \geq 1\end{cases}
$$

for all $(t, u) \in \mathbb{R} \times \mathbb{R}^{N}$. It is easy to check that $W(t, u)$ satisfies all conditions of Theorem 1.3, but it does not satisfy condition $\left(\mathrm{H}_{1}\right)$ of Theorem 1.1. Set

$$
W(t, u)= \begin{cases}\frac{1}{4}|u|, & |u|<1, \\ \frac{1}{2}|u|-\frac{u^{2}}{2\left(|u|+\ln |u|_{1}\right)}, & |u| \geq 1\end{cases}
$$


for all $(t, u) \in \mathbb{R} \times \mathbb{R}^{N}$. It is easy to check that $W(t, u)$ satisfies all conditions of Theorem 1.3, but it does not satisfy condition $\left(\mathrm{H}_{3}\right)$ of Theorem 1.1.

The remainder of this paper is organized as follows. In Section 2, some preliminary results are presented. In Section 3, we give the proof of Theorem 1.3.

\section{Preliminaries}

In this section, we give the variational setting for (1.1) and some related preliminary lemmas. Let $X:=H^{1}\left(\mathbb{R}, \mathbb{R}^{N}\right)$ be a Hilbert space with the inner product and the norm given, respectively, by

$$
\langle u, v\rangle_{X}=\int_{\mathbb{R}}[(\dot{u}(t), \dot{v}(t))+(u(t), v(t))] d t \quad \text { and } \quad\|u\|_{X}=\langle u, u\rangle_{X}^{\frac{1}{2}}, \quad \forall u, v \in X .
$$

We define an operator $K: X \rightarrow X$ by

$$
(K u, v)=\int_{\mathbb{R}}(B u(t), \dot{v}(t)) d t, \quad \forall u, v \in X .
$$

Since $B$ is an antisymmetric $N \times N$ constant matrix, $K$ is self-adjoint on $X$. Moreover, we denote by $J$ the self-adjoint extension of the operator $-\frac{d^{2}}{d t^{2}}+L(t)+K$ with the domain $D(J) \subset L^{2}\left(\mathbb{R}, \mathbb{R}^{N}\right)$.

Let $E:=D\left(|J|^{\frac{1}{2}}\right)$ be the domain of $|J|^{\frac{1}{2}}$, which is a Hilbert space equipped with the inner product and norm given by

$$
\begin{aligned}
& \langle u, v\rangle_{E}=\left(|J|^{\frac{1}{2}} u,|J|^{\frac{1}{2}} v\right)_{2}+(u, v)_{2}, \\
& \|u\|_{E}=\langle u, u\rangle_{E}^{\frac{1}{2}}
\end{aligned}
$$

for $u, v \in E$, where $(\cdot, \cdot)_{2}$ denotes the inner product in $L^{2}\left(\mathbb{R}, \mathbb{R}^{N}\right)$. Let $\|\cdot\|_{p}$ denote the usual norm on $L^{p}\left(\mathbb{R}, \mathbb{R}^{N}\right)(p \in[1, \infty])$.

Lemma 2.1 ([29]) Assume that $L$ satisfies $\left(\mathrm{L}_{1}\right)$ and $\left(\mathrm{L}_{2}\right)$. Then $E$ is compactly embedded in $L^{p}\left(\mathbb{R}, \mathbb{R}^{N}\right)$ for any $1 \leq p \leq \infty$.

By Lemma 2.1, the spectrum $\sigma(J)$ consists of eigenvalues numbered by $\eta_{1} \leq \eta_{2} \leq \cdots \leq$ $\eta_{k} \leq \cdots \rightarrow \infty$ (counted in their multiplicities) and a corresponding system of eigenfunctions $\left\{e_{k}\right\}\left(J e_{k}=\eta_{k} e_{k}\right)$ which forms an orthogonal basis in $L^{2}\left(\mathbb{R}, \mathbb{R}^{N}\right)$.

Set

$$
n^{-}=\sharp\left\{i \mid \eta_{i}<0\right\}, \quad n^{0}=\sharp\left\{i \mid \eta_{i}=0\right\}, \quad n^{+}=n^{-}+n^{0},
$$

and

$$
E^{-}=\operatorname{span}\left\{e_{1}, \ldots, e_{n^{-}}\right\}, \quad E^{0}=\operatorname{span}\left\{e_{n^{-}+1}, \ldots, e_{n^{0}}\right\}, \quad E^{+}=\mathrm{cl}_{E}\left(\operatorname{span}\left\{e_{n^{+}+1}, \ldots\right\}\right) .
$$

Thus, we have the orthogonal decomposition

$$
E=E^{-} \oplus E^{0} \oplus E^{+}
$$


with respect to the inner product $\langle\cdot, \cdot\rangle_{E}$. Now we introduce on $E$ the following inner product:

$$
\langle u, v\rangle=\left(|J|^{\frac{1}{2}} u,|J|^{\frac{1}{2}} v\right)_{2}+\left(u^{0}, v^{0}\right)_{2}
$$

and the norm

$$
\|u\|=\langle u, u\rangle^{\frac{1}{2}},
$$

where $u, v \in E$ with $u=u^{-}+u^{0}+u^{+}$and $v=v^{-}+v^{0}+v^{+}$. It is easy to verify that $\|\cdot\|_{E}$ and $\|\cdot\|$ are equivalent; see [8]. Evidently, the aforementioned decomposition is also orthogonal with respect to both inner products $(\cdot, \cdot)_{2}$ and $\langle\cdot, \cdot\rangle$.

Define the functional $\Psi$ on $E$ by

$$
\begin{aligned}
\Psi(u) & =\int_{\mathbb{R}}\left[\frac{1}{2}|\dot{u}(t)|^{2}+\frac{1}{2}(B u(t), \dot{u}(t))+\frac{1}{2}(L(t) u(t), u(t))-W(t, u(t))\right] d t \\
& =\frac{1}{2}\left\|u^{+}\right\|^{2}-\frac{1}{2}\left\|u^{-}\right\|^{2}-\int_{\mathbb{R}} W(t, u(t)) d t, \quad \forall u \in E .
\end{aligned}
$$

It follows from the assumptions that $\Psi$ is defined on $E$ and belongs to $C^{1}(E, \mathbb{R})$, and one can easily check that

$$
\Psi^{\prime}(u) v=\left\langle u^{+}, v^{+}\right\rangle-\left\langle u^{-}, v^{-}\right\rangle-\int_{\mathbb{R}}(\nabla W(t, u(t)), v(t)) d t
$$

for any $u, v \in E$ with $u=u^{-}+u^{0}+u^{+}$and $v=v^{-}+v^{0}+v^{+}$. Furthermore, it is routine to verify that any critical point of $\Psi$ in $E$ is a solution of system (1.1) with $u( \pm \infty)=0=\dot{u}( \pm \infty)$ (see $[20,21])$. In view of Lemma 2.1 , there exists $D_{p}>0$ such that

$$
\|u\|_{p} \leq D_{p}\|u\|, \quad \forall u \in E,
$$

where $p \in[1,+\infty]$.

Define $E_{j}=\mathbb{R} e_{j}$,

$$
Y_{k}=\bigoplus_{j=0}^{k} E_{j}, \quad Z_{k}=\overline{\bigoplus_{j=k}^{\infty} E_{j}}, \quad k \in \mathbb{N} .
$$

Lemma 2.2 Under assumptions $\left(\mathrm{L}_{1}\right)$ and $\left(\mathrm{L}_{2}\right)$, for $\varsigma \in[1,+\infty]$,

$$
\beta_{k}(\tau)=\sup _{u \in Z_{k},\|u\|=1}\|u\|_{\varsigma} \rightarrow 0 \quad \text { as } k \rightarrow \infty \text {. }
$$

Proof It is clear that $0<\beta_{k+1}(\varsigma) \leq \beta_{k}(\varsigma)$, so that $\beta_{k}(\varsigma) \rightarrow \tilde{\beta}(\varsigma), k \rightarrow \infty$. For every $k \geq 0$, there exists $u_{k} \in Z_{k}$ such that $\left\|u_{k}\right\|=1$ and $\left\|u_{k}\right\|_{\varsigma}>\frac{\beta_{k}}{2}$. For any $v \in X$, let $v=\sum_{i=1}^{\infty} \bar{b}_{i} e_{i}$, by the Cauchy-Schwartz inequality, one has

$$
\begin{aligned}
\left|\left\langle u_{k}, v\right\rangle\right| & =\left|\left\langle u_{k}, \sum_{i=1}^{\infty} \bar{b}_{i} e_{i}\right\rangle_{0}\right|=\left|\left\langle u_{k}, \sum_{i=k}^{\infty} \bar{b}_{i} e_{i}\right\rangle\right| \\
& \leq\left\|u_{k}\right\|\left\|\sum_{i=k}^{\infty} \bar{b}_{i} e_{i}\right\|=\sum_{i=k}^{\infty}\left\|\bar{b}_{i} e_{i}\right\| \rightarrow 0 \quad \text { as } k \rightarrow \infty,
\end{aligned}
$$


which implies that $u_{k} \rightarrow 0$. It follows from Lemma 2.1 that $u_{k} \rightarrow 0$ in $L^{q}\left(\mathbb{R}, \mathbb{R}^{N}\right)$. Thus we have proved that $\tilde{\beta}(\varsigma)=0$.

By Lemma 2.2, we can choose a positive integer $k_{0} \geq n^{+}+1$ such that

$$
\|u\|_{2}^{2} \leq \frac{1}{4 m_{0}}\|u\|^{2}
$$

where $m_{0}=\sup _{t \in \mathbb{R}}\left[\sup _{x \in \mathbb{R}^{N},|x|=1}(S(t) x, x)\right]$.

In order to prove our main result, we need the following lemma.

Lemma 2.3 ([8]) Let E be an infinite dimensional Banach space and $\Phi \in C^{1}(E, \mathbb{R})$ be even, satisfy the (PS) condition, and $\Psi(0)=0$. If $X=Y \oplus Z$, where $Y$ is finite dimensional, and $\Psi$ satisfies

$\left(\mathrm{S}_{1}\right) \Psi$ is bounded from below on $Z$;

$\left(\mathrm{S}_{2}\right)$ for each finite dimensional subspace $\widetilde{E} \subset E$, there are positive constants $\rho=\rho(\widetilde{E})$ and $\alpha=\alpha(\widetilde{E})$ such that $\left.\Psi\right|_{B_{\rho} \cap \widetilde{E}} \leq 0$ and $\left.\Psi\right|_{\partial B_{\rho} \cap \widetilde{E}} \leq-\alpha$, where $B_{\rho}=\{x \in E:\|x\| \leq \rho\}$.

Then $\Psi$ possesses infinitely many nontrivial critical points.

Remark 2.1 As shown in [34], a deformation lemma can be proved with condition $(C)$ replacing $(P S)$ condition, and it turns out that Lemma 2.3 holds true under condition $(C)$. We say that $\Psi$ satisfies condition $(C)$, i.e. for any $\left\{u_{n}\right\} \subset E,\left\{u_{n}\right\}$ has a convergent subsequence if $\Psi\left(u_{n}\right)$ is bounded and $\left(1+\left\|u_{n}\right\|\right)\left\|\Psi^{\prime}\left(u_{n}\right)\right\| \rightarrow 0$ as $n \rightarrow \infty$.

\section{Proof of Theorem 1.3}

Set $Y=Y_{k_{0}}, Z=Z_{k_{0}}$.

Lemma 3.1 Suppose that $\left(\mathrm{W}_{1}\right)$ and $\left(\mathrm{W}_{2}\right)$ are satisfied. Then $\Psi$ is bounded from below on $Z$.

Proof By virtue of $\left(\mathrm{W}_{1}\right),\left(\mathrm{W}_{2}\right),(2.3),(2.5)$, and (2.8), we have

$$
\begin{aligned}
\Psi(u) & =\frac{1}{2}\left\|u^{+}\right\|^{2}-\frac{1}{2}\left\|u^{-}\right\|^{2}-\sum_{n \in \mathbb{Z}} W(n, u(n)) \\
& =\frac{1}{2}\|u\|^{2}-\sum_{n \in \mathbb{Z}} W(n, u(n)) \\
& \geq \frac{1}{2}\|u\|^{2}-m_{0}\|u\|_{2}^{2}-\bar{a}\|u\|_{1}-\bar{b}\|u\|_{v+1}^{v+1} \\
& \geq \frac{1}{4}\|u\|^{2}-\bar{a} D_{1}\|u\|-\bar{b} D_{v+1}^{v+1}\|u\|^{v+1} \rightarrow+\infty
\end{aligned}
$$

as $\|u\| \rightarrow \infty$ and $u \in Z_{k_{0}}$. The proof is completed.

Lemma 3.2 Assume that $\left(\mathrm{H}_{4}\right)$ holds. Then for each finite dimensional subspace $\widetilde{E} \subset E$, there are positive constants $\rho=\rho(\widetilde{E})$ and $\alpha=\alpha(\widetilde{E})$ such that $\left.\Psi\right|_{B_{\rho} \cap \widetilde{E}} \leq 0$ and $\left.\Psi\right|_{\partial B_{\rho} \cap \widetilde{E}} \leq-\alpha$, where $B_{\rho}=\{x \in E:\|x\| \leq \rho\}$. 
Proof Let $\widetilde{E} \subset E$ be any finite dimensional subspace. Then there exists $M_{0}>0$ such that

$$
\|u\|^{2} \leq M_{0}\|u\|_{2}^{2}, \quad \forall u \in \widetilde{E} .
$$

By virtue of $\left(\mathrm{H}_{4}\right)$, for $M_{0}$ given above, there exists a constant $\sigma>0$,

$$
W(t, u) \geq M_{0}|u|^{2}, \quad \forall t \in \mathbb{R} \text { and }|u| \leq \sigma .
$$

In view of (2.8), for any $u \in \widetilde{E}$ with $\|u\| \leq \frac{\sigma}{D_{\infty}}$, we have

$$
\|u\|_{\infty} \leq \sigma
$$

By (3.2)-(3.4), we have

$$
\begin{aligned}
\Psi(u) & =\frac{1}{2}\left\|u^{+}\right\|^{2}-\frac{1}{2}\left\|u^{-}\right\|^{2}+\int_{\mathbb{R}} W(t, u(t)) d t \\
& \leq \frac{1}{2}\left\|u^{+}\right\|^{2}-\int_{\mathbb{R}} W(t, u(t)) d t \\
& \leq \frac{1}{2}\left\|u^{+}\right\|^{2}-M_{0}\|u\|_{2}^{2} \\
& \leq-\frac{1}{2}\|u\|^{2}
\end{aligned}
$$

for any $u=u^{-}+u^{0}+u^{+} \in \widetilde{E}$ with $\|u\| \leq \frac{\sigma}{D_{\infty}}$. Then there exist $\rho=\rho(\widetilde{E})>0$ and $\alpha=\alpha(\widetilde{E})>0$ such that

$$
\Psi(u) \leq 0, \quad \forall u \in B_{\rho} \cap \widetilde{E} ; \quad \Psi(u) \leq-\alpha, \quad \forall u \in \partial B_{\rho} \cap \widetilde{E} .
$$

The proof is completed.

Lemma 3.3 Under the assumptions of Theorem 1.3, $\Psi$ satisfies condition $(C)$.

Proof Let $\left\{u_{n}\right\} \subset E$ is a $(C)$ sequence of $\Psi$, that is, $\left\{\Psi\left(u_{n}\right)\right\}$ is bounded and

$$
\left(1+\left\|u_{n}\right\|\right)\left\|\Psi^{\prime}\left(u_{n}\right)\right\| \rightarrow 0 \quad \text { as } n \rightarrow \infty
$$

then there exists a constant $M_{1}>0$ such that

$$
\left|\Phi\left(u_{m}\right)\right| \leq M_{1}, \quad\left(1+\left\|u_{n}\right\|\right)\left\|\Psi^{\prime}\left(u_{n}\right)\right\| \leq M_{1}
$$

for every $n \in \mathbb{N}$. We choose $k \geq n^{+}+1$ large enough such that

$$
\|u\|^{2} \geq 2 m_{0}\|u\|_{2}^{2}, \quad \forall u \in Z_{k}
$$

where $m_{0}=\sup _{t \in \mathbb{R}}\left[\sup _{x \in \mathbb{R}^{N},|x|=1}(S(t) x, x)\right]$. We now prove that $\left\{u_{n}\right\}$ is bounded in $E$. In fact, if not, we may assume by contradiction that $\left\|u_{n}\right\| \rightarrow \infty$ as $n \rightarrow \infty$. Let $u_{n}=\tilde{w}_{n}+\tilde{v}_{n}, z_{n}=$ $\frac{u_{n}}{\left\|u_{n}\right\|}$, then $\left\|z_{n}\right\|=1, z_{n}=w_{n}+v_{n} \in E$, where $w_{n}=\frac{\tilde{w}_{n}}{\left\|u_{n}\right\|}, v_{n}=\frac{\tilde{v}_{n}}{\left\|u_{n}\right\|}, \tilde{w}_{n} \in Y_{k}, \tilde{v}_{n} \in Z_{k}$. After passing to a subsequence, we have $z_{n} \rightarrow z, w_{n} \rightarrow w$, and $\gamma=\lim _{n \rightarrow \infty}\left\|v_{n}\right\|$ exists. 
Case 1. $\gamma=0$. Since $\operatorname{dim} Y_{k}<\infty$, we obtain $\left\|w_{n}\right\| \rightarrow\|w\|=1$. It follows from (3.7) that

$$
\frac{3}{2} M_{1} \geq \Psi\left(u_{n}\right)-\frac{1}{2} \Psi^{\prime}\left(u_{n}\right) u_{n} \geq \int_{\mathbb{R}}\left(W\left(t, u_{n}(t)\right)-\frac{1}{2}\left(\nabla W\left(t, u_{n}(t)\right), u_{n}(t)\right)\right) d t .
$$

By virtue of $\left(\mathrm{H}_{6}\right)$, for any $\eta>0$, there exists $M_{2}>0$ such that

$$
\widetilde{W}(t, u)=W(t, u)-\frac{1}{2}(\nabla W(t, u), u) \geq \eta, \quad \forall t \in \mathbb{R},|u| \geq M_{2}
$$

For any $\varepsilon>0$, define $\Lambda_{\varepsilon}:=\{t \in \mathbb{R}:|w(t)| \geq \varepsilon\}$ and $\Lambda_{n \varepsilon}:=\left\{t \in \mathbb{R}:\left|v_{n}(t)\right| \geq \frac{\varepsilon}{2}\right\}$. First, we claim that there exists $\varepsilon_{0}>0$ such that

$$
\operatorname{meas}\left\{t \in \mathbb{R}:|u(t)| \geq \varepsilon_{0}\right\} \geq \varepsilon_{0}, \quad \forall u \in Y_{k} \text { with }\|u\|=1 \text {. }
$$

Otherwise, for any positive integer $m$, there exists $w_{m} \in Y_{k}$ with $\left\|w_{m}\right\|=1$ such that

$$
\operatorname{meas}\left\{t \in \mathbb{R}:\left|w_{m}(t)\right| \geq \frac{1}{m}\right\}<\frac{1}{m} \text {. }
$$

Passing to a subsequence if necessary, we may assume $w_{m} \rightarrow w_{0}$ in $E$ for some $w_{0} \in Y_{k}$ since $\operatorname{dim} Y_{k}<\infty$. Evidently, $\left\|w_{0}\right\|=1$. By the equivalence of the norms on the finite dimensional space $Y_{k}$, we have

$$
\int_{\mathbb{R}}\left|w_{m}(t)-w_{0}(t)\right|^{2} d t \rightarrow 0 \quad \text { as } m \rightarrow \infty
$$

Thus there exists $\varepsilon_{1}>0$ such that

$$
\operatorname{meas}\left\{t \in \mathbb{R}:\left|w_{0}(t)\right| \geq \varepsilon_{1}\right\} \geq \varepsilon_{1} \text {. }
$$

In fact, if not, then, for all positive integers $m$, we have

$$
\operatorname{meas}\left\{t \in \mathbb{R}:\left|w_{0}(t)\right| \geq \frac{1}{m}\right\}=0
$$

It implies that

$$
0 \leq \int_{\mathbb{R}}\left|w_{0}(t)\right|^{4} d t<\frac{1}{m^{2}}\left\|w_{0}\right\|_{2}^{2} \rightarrow 0 \quad \text { as } m \rightarrow \infty .
$$

Hence $w_{0}=0$, which contradicts that $\left\|w_{0}\right\|_{0}=1$. Thus, (3.13) holds.

Now set

$$
\Upsilon_{0}=\left\{t \in \mathbb{R}:\left|w_{0}(t)\right| \geq \varepsilon_{1}\right\}, \quad \Upsilon_{m}=\left\{t \in \mathbb{R}:\left|w_{m}(t)\right|<\frac{1}{m}\right\},
$$

and $\Upsilon_{m}^{c}=\mathbb{R} \backslash \Upsilon_{m}=\left\{t \in \mathbb{R}:\left|w_{m}(t)\right| \geq \frac{1}{m}\right\}$. By virtue of (3.11) and (3.13), we obtain

$$
\begin{aligned}
\operatorname{meas}\left(\Upsilon_{m} \cap \Upsilon_{0}\right) & =\operatorname{meas}\left(\Upsilon_{0} \backslash \Upsilon_{m}^{c} \cap \Upsilon_{0}\right) \\
& \geq \operatorname{meas}\left(\Upsilon_{0}\right)-\operatorname{meas}\left(\Upsilon_{m}^{c} \cap \Upsilon_{0}\right) \\
& \geq \varepsilon_{1}-\frac{1}{m}
\end{aligned}
$$


for all positive integers $m$. Let $m$ be large enough such that $\frac{1}{2} \varepsilon_{1}-\frac{1}{m}>\frac{1}{4} \varepsilon_{1}$. Thus, for $m$ large enough,

$$
\begin{aligned}
\int_{\mathbb{R}}\left|w_{m}(t)-w_{0}(t)\right|^{2} d t & \geq \int_{t \in \Upsilon_{0} \cap \Upsilon_{m}}\left|w_{m}(t)-w_{0}(t)\right|^{2} d t \\
& \geq \frac{1}{2} \int_{t \in \Upsilon_{0} \cap \Upsilon_{m}}\left|w_{0}(t)\right|^{2} d t-\int_{t \in \Upsilon_{0} \cap \Upsilon_{m}}\left|w_{m}(t)\right|^{2} d t \\
& \geq\left(\frac{1}{2} \varepsilon_{1}-\frac{1}{m}\right) \operatorname{meas}\left(\Upsilon_{m} \cap \Upsilon_{0}\right) \\
& \geq \frac{\varepsilon_{1}^{2}}{16}>0,
\end{aligned}
$$

which is a contradiction to (3.12). Thus, there exists $\varepsilon>0$ such that meas $\left(\Lambda_{\varepsilon}\right) \geq \varepsilon$.

In view of (2.5), we obtain

$$
\operatorname{meas}\left(\Lambda_{n \varepsilon}\right) \leq \frac{4}{\varepsilon^{2}} \int_{\mathbb{R}}\left|v_{n}(t)\right|^{2} d t \leq \frac{4 D_{2}^{2}}{\varepsilon^{2}}\left\|v_{n}\right\|^{2} \rightarrow 0 \quad \text { as } n \rightarrow \infty
$$

Then we have meas $\left(\Lambda_{\varepsilon} \backslash \Lambda_{n \varepsilon}\right) \rightarrow$ meas $\left(\Lambda_{\varepsilon}\right)$ as $n \rightarrow \infty$. Therefore, there exists $N_{0}>0$ such that $\left|z_{n}(t)\right| \geq \frac{\varepsilon}{3}, \forall t \in \Lambda_{\varepsilon} \backslash \Lambda_{n \varepsilon}$ and $n \geq N_{0}$, then we have $\left|u_{n}(t)\right| \geq \frac{\varepsilon}{3}\left\|u_{n}\right\|, \forall t \in \Lambda_{\varepsilon} \backslash \Lambda_{n \varepsilon}$ and $n \geq N_{0}$. By $\left(\mathrm{W}_{3}\right),(3.9)$, and (3.10), there exists $N_{1}>0$ such that

$$
\frac{3}{2} M_{1} \geq \int_{\mathbb{R}} \tilde{W}\left(t, u_{n}(t)\right) d t \geq \int_{t \in \Lambda_{\varepsilon} \backslash \Lambda_{n \varepsilon}} \eta d t \geq \eta \operatorname{meas}\left(\Lambda_{\varepsilon} \backslash \Lambda_{n \varepsilon}\right), \quad \forall n \geq N_{1},
$$

which gives a contradiction due to the arbitrariness of $\eta$.

Case 2. $\gamma>0$. In view of $\left(\mathrm{W}_{1}\right),\left(\mathrm{W}_{2}\right),(2.5),(3.7),(3.8)$, and Hölder's inequality, we get

$$
\begin{aligned}
M_{1} & \geq \Psi^{\prime}\left(u_{n}\right) \tilde{v}_{n}=\left\|\tilde{v}_{n}\right\|^{2}-\int_{\mathbb{R}}\left(\nabla W\left(t, u_{n}(t)\right), \tilde{v}_{n}(t)\right) d t \\
& \geq\left\|\tilde{v}_{n}\right\|^{2}-\int_{\mathbb{R}}\left[\left(S(t) u_{n}(t), \tilde{v}_{n}(t)\right)+\left(\bar{a}+\bar{b}\left|u_{n}(t)\right|^{v}\right)\left|\bar{v}_{n}(t)\right|\right] d t \\
& \geq\left\|\tilde{v}_{n}\right\|^{2}-m_{0}\left\|\tilde{v}_{n}\right\|_{2}^{2}-\bar{a}\left\|\tilde{v}_{n}\right\|_{1}-\bar{b}\left\|u_{n}\right\|_{2 v}^{v}\left\|\tilde{v}_{n}\right\|_{2} \\
& \geq \frac{1}{2}\left\|\tilde{v}_{n}\right\|^{2}-\bar{a} D_{1}\left\|\tilde{v}_{n}\right\|-\bar{b} D_{2} D_{2 v}^{v}\left\|u_{n}\right\|^{v}\left\|\tilde{v}_{n}\right\| \\
& \geq \frac{1}{2}\left\|\tilde{v}_{n}\right\|^{2}-\bar{a} D_{1}\left\|u_{n}\right\|-\bar{b} D_{2} D_{2 v}^{v}\left\|u_{n}\right\|^{v+1} .
\end{aligned}
$$

Dividing by $\left\|u_{n}\right\|^{2}$ on both sides of (3.15), we obtain

$$
0 \geq \frac{\gamma^{2}}{2}>0
$$

which gives a contradiction.

Thus, $\left\{u_{n}\right\}$ is bounded. Next, we show that $\left\{u_{n}\right\}$ has a convergent subsequence. In view of the boundedness of $\left\{u_{n}\right\}$, without loss of generality, we may assume that

$$
u_{n} \rightarrow u, \quad u_{n}^{+} \rightarrow u^{+}, \quad u_{n}^{-} \rightarrow u^{-}, \quad u_{n}^{0} \rightarrow u^{0} .
$$


It follows from (2.4) that

$$
\begin{aligned}
\left\|u_{n}^{+}-u^{+}\right\|^{2}= & \left(\Psi^{\prime}\left(u_{n}\right)-\Psi^{\prime}(u)\right)\left(u_{n}^{+}-u^{+}\right) \\
& +\int_{\mathbb{R}}\left(\nabla W\left(t, u_{n}\right)-\nabla W(t, u), u_{n}^{+}-u^{+}\right) d t .
\end{aligned}
$$

It is clear that

$$
\left(\Psi^{\prime}\left(u_{n}\right)-\Psi^{\prime}(u)\right)\left(u_{n}^{+}-u^{+}\right) \rightarrow 0 \quad \text { as } n \rightarrow \infty
$$

By (2.5), Lemma 2.1, $\left(\mathrm{W}_{1}\right),\left(\mathrm{W}_{2}\right)$, and Hölder's inequality, we have

$$
\begin{aligned}
& \int_{\mathbb{R}}\left(\nabla W\left(t, u_{n}\right)-\nabla W(t, u), u_{n}^{+}-u^{+}\right) d t \\
& \leq \bar{a}\left\|u_{n}^{+}-u^{+}\right\|_{1}+\left(m_{0}\left\|u_{n}\right\|_{2}+\bar{b}\left\|u_{n}\right\|_{2 v}^{v}\right)\left\|u_{n}^{+}-u^{+}\right\|_{2} \\
&+\bar{a}\left\|u_{n}^{+}-u^{+}\right\|_{1}+\left(m_{0}\|u\|_{2}+\bar{b}\|u\|_{2 v}^{v}\right)\left\|u_{n}^{+}-u^{+}\right\|_{2} \\
& \leq 2 \bar{a}\left\|u_{n}^{+}-u^{+}\right\|_{1}+\left(m_{0} D_{2}\left\|u_{n}\right\|+\bar{b} D_{2 v}^{v}\left\|u_{n}\right\|^{v}\right)\left\|u_{n}^{+}-u^{+}\right\|_{2} \\
&+\left(m_{0} D_{2}\|u\|+\bar{b} D_{2 v}^{v}\|u\|^{v}\right)\left\|u_{n}^{+}-u^{+}\right\|_{2} \rightarrow 0
\end{aligned}
$$

as $n \rightarrow \infty$. Therefore, by (3.17)-(3.19), we get $\left\|u_{m}^{+}-u^{+}\right\| \rightarrow 0$ as $n \rightarrow \infty$. Consequently, $u_{n} \rightarrow u$. The proof is completed.

Proof of Theorem 1.3 Obviously, $\Psi \in C^{1}(X, \mathbb{R})$ is even and $\Phi(0)=0$. It follows from Lemmas 3.1-3.3 that all conditions of Lemma 2.3 are satisfied. By Lemma 2.3, we see that $\Psi$ possesses infinitely many nontrivial critical points, that is, system (1.1) possesses infinitely many nontrivial homoclinic orbits.

\section{Competing interests}

The authors declare that they have no competing interests.

\section{Authors' contributions}

All authors contributed equally to the writing of this paper. All authors read and approved the final manuscript.

\section{Author details}

${ }^{1}$ School of Mathematics and Physics, University of South China, Hengyang, Hunan 421001, China. ${ }^{2}$ School of Mathematics and Statistics, Central South University, Changsha, Hunan 410083, China.

\section{Acknowledgements}

The first author was supported by the Doctor Priming Fund Project of University of South China (2014XQD13) and National Natural Science Foundation of China (Grants Nos. 11526111 and 11501284).

Received: 24 October 2015 Accepted: 8 March 2016 Published online: 16 March 2016

\section{References}

1. Poincaré, H: Les Méthodes Nouvelles de la Mécanique Céleste. Gauthier-Villars, Paris (1897-1899)

2. Alves, $\mathrm{CO}$, Carriao, PC, Miyagaki, OH: Existence of homoclinic orbits for asymptotically periodic systems involving Duffing-like equation. Appl. Math. Lett. 16, 639-642 (2003)

3. Caldiroli, P, Montecchiari, P: Homoclinic orbits for second order Hamiltonian systems with potential changing sign. Commun. Appl. Nonlinear Anal. 1(2), 97-129 (1994)

4. Carriao, PC, Miyagaki, OH: Existence of homoclinic solutions for a class of time-dependent Hamiltonian systems. J. Math. Anal. Appl. 291, 203-213 (2004)

5. Chen, $\mathrm{H}, \mathrm{He}, \mathrm{Z}$ : Infinitely many homoclinic solutions for a class of second-order Hamiltonian systems. Adv. Differ. Equ. 2014, 161 (2014)

6. Coti Zelati, V, Rabinowitz, PH: Homoclinic orbits for second order Hamiltonian systems possessing superquadratic potentials. J. Am. Math. Soc. 4, 693-727 (1991) 
7. Ding, Y, Lee, C: Homoclinics for asymptotically quadratic and superquadratic Hamiltonian systems. Nonlinear Anal. 71, 1395-1413 (2009)

8. Ding, Y: Existence and multiplicity results for homoclinic solutions to a class of Hamiltonian systems. Nonlinear Anal. 25, 1095-1113 (1995)

9. Izydorek, M, Janczewska, J: Homoclinic solutions for a class of the second order Hamiltonian systems. J. Differ. Equ. 219, 375-389 (2005)

10. Korman, P, Lazer, AC: Homoclinic orbits for a class of symmetric Hamiltonian systems. Electron. J. Differ. Equ. 1994, 1 (1994)

11. LV, X, Lu, S, Jiang, J: Homoclinic solutions for a class of second-order Hamiltonian systems. Nonlinear Anal., Real World Appl. 13, 176-185 (2012)

12. Omana, W, Willem, M: Homoclinic orbits for a class of Hamiltonian systems. Differ. Integral Equ. 5, 1115-1120 (1992)

13. Paturel, E: Multiple homoclinic orbits for a class of Hamiltonian systems. Calc. Var. Partial Differ. Equ. 12, 117-143 (2001)

14. Rabinowitz, PH: Homoclinic orbits for a class of Hamiltonian systems. Proc. R. Soc. Edinb. A 114, 33-38 (1990)

15. Rabinowitz, PH, Tanaka, K: Some results on connecting orbits for a class of Hamiltonian systems. Math. Z. 206, 473-499 (1991)

16. Salvatore, A: Homoclinic orbits for a special class of nonautonomous Hamiltonian systems. Nonlinear Anal. 30 4849-4857 (1997)

17. Sun, J, Chen, H, Nieto, JJ: Homoclinic solutions for a class of subquadratic second-order Hamiltonian systems. J. Math. Anal. Appl. 373, 20-29 (2011)

18. Tang, X, Lin, X: Homoclinic solutions for a class of second-order Hamiltonian systems. J. Math. Anal. Appl. 354 539-549 (2009)

19. Tang, X, Lin, X: Existence of infinitely many homoclinic orbits in Hamiltonian systems. Proc. R. Soc. Edinb. A 141 1103-1119 (2011)

20. Wang, L, Tang, C: Existence and multiplicity of homoclinic orbits for second order Hamiltonian systems without (AR) condition. Discrete Contin. Dyn. Syst., Ser. B 15, 255-271 (2011)

21. Wang, J, Xu, J, Zhang, F: Homoclinic orbits for a class of Hamiltonian systems with superquadratic or asymptotically quadratic potentials. Commun. Pure Appl. Anal. 10, 269-286 (2011)

22. Yang, J, Zhang, F: Infinitely many homoclinic orbits for the second-order Hamiltonian systems with super-quadratic potentials. Nonlinear Anal., Real World Appl. 10, 1417-1423 (2009)

23. Zhang, Q, Liu, C: Infinitely many homoclinic solutions for second order Hamiltonian systems. Nonlinear Anal. TMA 72 894-903 (2010)

24. Zou, W, Li, S: Infinitely many homoclinic orbits for the second-order Hamiltonian systems. Appl. Math. Lett. 16, 1283-1287 (2003)

25. Chen, G: Non-periodic damped vibration systems with sublinear terms at infinity: infinitely many homoclinic orbits. Nonlinear Anal. 92, 168-176 (2013)

26. Chen, G: Nonperiodic damped vibration systems with asymptotically quadratic terms at infinity: infinitely many homoclinic orbits. Abstr. Appl. Anal. 2013, Article ID 937128 (2013)

27. Chen, $\mathrm{H}, \mathrm{He}, \mathrm{Z}$ : New existence and multiplicity of homoclinic solutions for second order non-autonomous systems. Electron. J. Qual. Theory Differ. Equ. 2014, 22 (2014)

28. Chen, P, Tang, X: Infinitely many homoclinic solutions for a class of damped vibration problems. Math. Methods Appl. Sci. 37, 2297-2307 (2014)

29. Sun, J, Nieto, JJ, Otero-Novoa, M: On homoclinic orbits for a class of damped vibration systems. Adv. Differ. Equ. 2012, $102(2012)$

30. Yuan, R, Zhang, Z: Homoclinic solutions for a class of second order non-autonomous systems. Electron. J. Differ. Equ. 2009, $128(2009)$

31. Zhang, Z, Yuan, R: Homoclinic solutions of some second order non-autonomous systems. Nonlinear Anal. 71, 5790-5798 (2009)

32. Wu, X, Zhang, W: Existence and multiplicity of homoclinic solutions for a class of damped vibration problems. Nonlinear Anal. 74, 4392-4398 (2011)

33. Zhu, W: Existence of homoclinic solutions for a class of second order systems. Nonlinear Anal. 75, 2455-2463 (2012)

34. Bartolo, P, Benci, V, Fortunato, D: Abstract critical point theorems and applications to some nonlinear problems with 'strong' resonance at infinity. Nonlinear Anal. 7, 981-1012 (1983)

\section{Submit your manuscript to a SpringerOpen ${ }^{\circ}$ journal and benefit from:}

- Convenient online submission

Rigorous peer review

- Immediate publication on acceptance

Open access: articles freely available online

- High visibility within the field

- Retaining the copyright to your article 